\title{
Research Paper \\ Evaluation of Antioxidant and Anticancer Effects of Nanoemulsions Prepared Using Dill Essential Oil
}

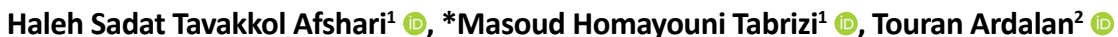

1. Department of Biology, Faculty of Science, Mashhad Branch, Islamic Azad University, Mashhad, Iran.

2. Department of Chemistry, Faculty of Science, Mashhad Branch, Islamic Azad University, Mashhad, Iran.

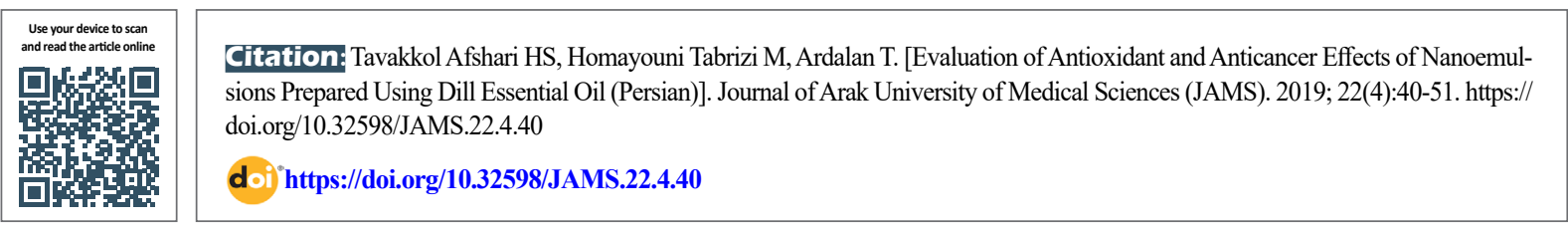

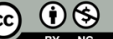

Article Info:

Received: 10 Jun 2019

Accepted: 14 Aug 2019

Available Online: 01 Oct 2019

Key words:

Nanoemulsion, Dill,

Antioxidant, Anti-

cancer

\section{A B STRACT}

Background and Aim Among nano-pharmaceutical materials, nanoemulsions are an important tool in the field of nanotechnology. They have been designed for clinical and therapeutic applications. Since the therapeutic efficacy of cancer is measured by the ability of the drug to reduce and eliminate tumors without damaging healthy tissues, nanoemulsions can be useful as a targeted drug carrier. Therefore, we are going to study the antioxidant and anticancer effects of nanoemulsions prepared using dill essential oil. Methods and Materials Evaluate the antioxidant properties, we used DPPH (1,1-diphenyl-2-picrylhydrazyl) and ABTS (2,2'-azino-bis[3-ethylbenzothiazoline-6-sulphonic acid]) tests. We also applied MTT assay for the evaluation of induced cytotoxicity in liver hepatocellular carcinoma cells and normal human umbilical vein endothelial cells.

Ethical Considerations The Research Ethics Committee of Islamic Azad University (Mashhad Branch) approved this study (Code: IR.IAU.MSHD.REC.1398.027).

Results Nanoemulsions prepared using dill essential oil has a good potential for inhibiting DPPH (IC50=500 $\mu \mathrm{g} / \mathrm{mL}$ ) and ABTS (IC50 $=420 \mu \mathrm{g} / \mathrm{mL}$ ) radicals. Nanoemulsions also caused a little toxicity to both cell lines. Nanoemulsions reduced cell viability in a dose-dependent manner and the cytotoxicity induced to cancer cells was higher than normal cells.

Conclusion The present study indicates that nanoemulsions prepared by dill essential oil will have the potential to become a therapeutic strategy for diseases caused by oxidative stress.

\section{Extended Abstract}

\section{Introduction}

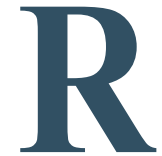

esearchers have shown interest in using nanotechnology for the treatment of cancer in recent years. This technology presents unique ways to predict, prevent, or treat cancer at an early stage [1]. Since nanoparticles are 100 to 1000 times smaller than cancer cells, they can interact with tumor-specific proteins at the surface or within cancer cells and act as carriers of drug delivery into these cells [2]. As the efficacy of cancer treatment depends on the drug's potential to reduce and eliminate tumors without damaging healthy tissue, nanoemulsion as a targeted drug carrier can be useful in this area [8]. The anticancer properties of medicinal plants to inhibit cancer progression and induce apoptosis in cancer cells have

\section{* Corresponding Author:}

Masoud Homayouni Tabrizi, PhD.

Address: Department of Biology, Faculty of Science, Mashhad Branch, Islamic Azad University, Mashhad, Iran.

Tel: +98 (915) 4505894

E-mail: mhomayouni6@gmail.com 
been extensively investigated in many studies [16]. One of the medicinal plants is Anethum graveolens L. (dill), which has anticancer potential, and its effects have been reported in the treatment of cancers, including liver and cervical cancers [13]. This study aims to assess the antioxidant effects of nanoemulsion synthesized by the essential oil of dill and evaluate its cytotoxicity on HepG2 cell line in vitro.

\section{Materials and Methods}

Stable nanoemulsion derived from the dill essential oil was prepared using ultrasonic waves at $200 \mathrm{~W}$ for $30 \mathrm{~min}$. Liver cancer cell lines (HepG2) and human umbilical vein endothelial cells (HUVECs) were cultured in 96-well plates in sterile conditions and kept in the incubator at $37^{\circ} \mathrm{C}, 5 \%$ $\mathrm{CO}_{2}$, and $95 \%$ humidity. The plates with a cell count of about 5000 per well were used to evaluate the toxicity of the drugs. This evaluation was performed using the MTT assay [17]. To evaluate the antioxidant activities of nanoemulsions, we ran two tests of DPPH (1,1-diphenyl-2-picrylhydrazyl) and ABTS (2,2-azino-bis[3-ethylbenzothiazoline-6-sulfonic acid]) [18]. The viability of treated cells and the antioxidant activity of plant-derived nanoemulsion were analyzed in SPSS V. 22 by using one-way Analysis of Variance (ANOVA) and the Least Significant Difference (LSD) methods.

\section{Results}

The plant-derived nanoemulsion had a toxic effect on both cell lines, and this effect was dose-dependent. In other words, with increasing concentrations of this substance, the survival of both cell lines decreased but its impact on HepG2 was more significant than on HUVEC. Evaluation of the antioxidant effect of nanoemulsion using DPPH and

DPPH

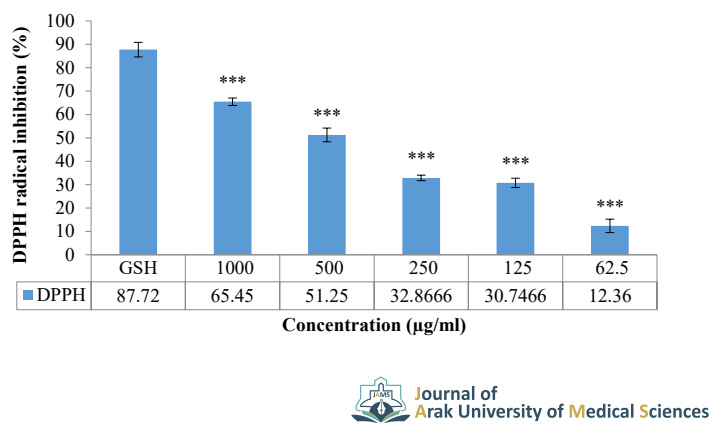

Figure 1. DPPH test results in evaluating the antioxidant activity of dill essential oil-based nanoemulsion. In this test, glutathione was used as a standard.

**Significant at $\mathrm{P}<0.001$
ABTS tests showed that it had antioxidant activity, and its antioxidant activity increased by increasing its concentration. DPPH test results reported that about $500 \mu \mathrm{g} / \mathrm{mL}$ of nanoemulsion derived from dill plant could remove 50\% of free radicals (Figure 1). ABTS test results indicated that $420 \mu \mathrm{g} / \mathrm{mL}$ of nanoemulsion could eliminate $50 \%$ of released free radicals in the environment (Figure 2).

\section{Discussion}

The results of this study showed that by increasing the concentration of nanoemulsion synthesized from dill essential oil, the percentage of free radicals inhibition increased. In other words, the antioxidant property of this substance is dose-dependent. The results of MTT assay on HepG2 and normal HUVEC cell lines showed that this substance had toxic effects on both cancerous and healthy cells but had a more poisonous effect on HepG2 than on HUVEC. The toxic effects of prepared nanoemulsion on the cells were dose-dependent; as its dose increases, its toxic effects also increase, and cell viability decreases.

Recently, new nanotechnology-based treatment strategies have emerged as an alternative to chemotherapy. Among different types of nanoproducts, nanoemulsions have several advantages for the delivery of anticancer drugs, which can increase the intracellular concentration of the drugs and thereby reducing the cytotoxicity of chemotherapy [21]. Various studies have shown that dill has multiple compounds such as carvone, limonene, and phellandrene, and its antioxidant and cytotoxic properties can be attributed to them [22].

In our study, dill essential oil-based nanoemulsion had potent antioxidant activity. This property indicates the poten-

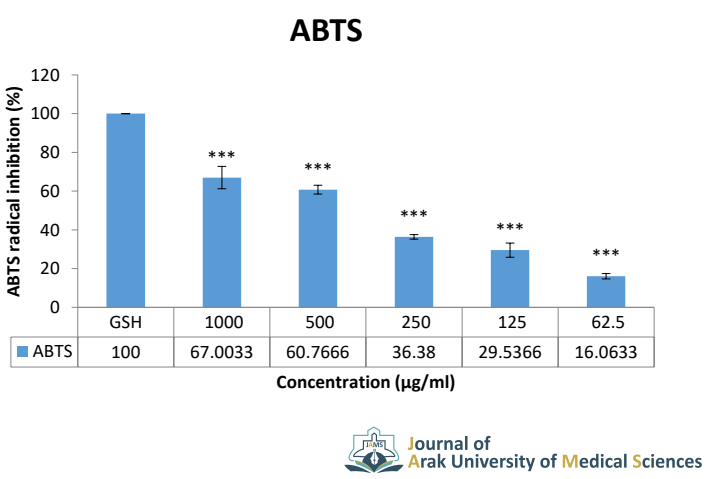

Figure 2. ABTS test results of evaluating the antioxidant activity of dill essential oil-based nanoemulsion. In this test, glutathione was used as a standard.

**Significant at $\mathrm{P}<0.001$ 
tial of this nanoemulsion to become a therapeutic strategy for future diseases caused by oxidative stress and cancer. It is recommended to evaluate effective toxicity on other cancer cell lines.

\section{Ethical Considerations}

Compliance with ethical guidelines

All experiments in this study were according to the ethical guidelines of the Ethics Committee of Islamic Azad University of Mashhad (ethical code: IR.IAU.MSHD. REC.1398.02.7).

\section{Funding}

This study was extracted from a research proposal approved by the Ethics Committee of Mashahd University of Medical Sciences (Code: IR.IAU.MSHD.REC.1398.027)

\section{Authors' contributions}

Conceptualization, visualization, supervision, project administration, and funding by Masoud Homayouni Tabrizi; methodology and validation by Masoud Homayouni Tabrizi and Touran Ardalan; Analysis and investigation by Haleh Sadat Tavakkol Afshari; and resources by all authors.

Conflicts of interest

The authors declare no conflict of interest. 


\title{
بررسى خصوصيات آنتى اكسيدانى وضدسرطانى نانوامولسيون سنتزشده توسط اسانس تياه شويد
}

\author{
هاله سادات توكل افشارى' (1)، •مسعود همايونى تبريزى' (1)، توران اردلان \\ 1- كروه زيستشئاسى، دانشكده علوم يايه، واحد مشهد، دانشكاه آزاد اسلامى، مشهيده ايران.

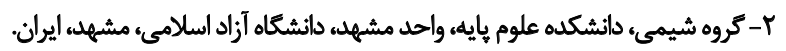

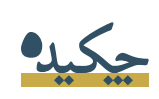

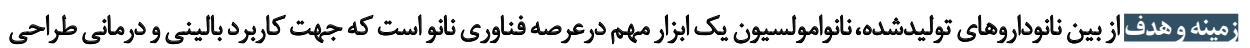

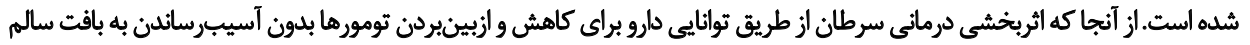

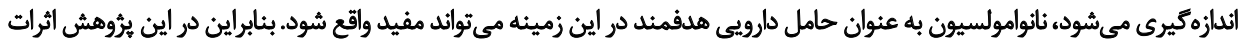

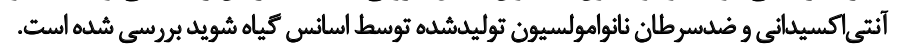

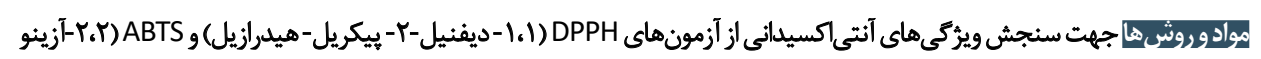

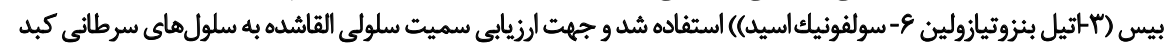

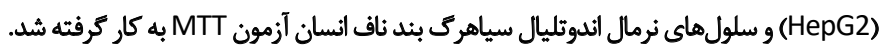

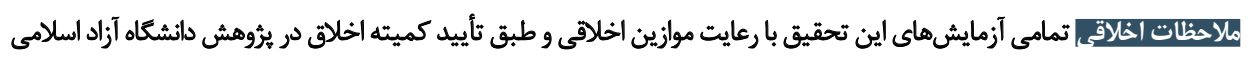

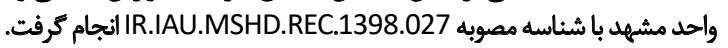

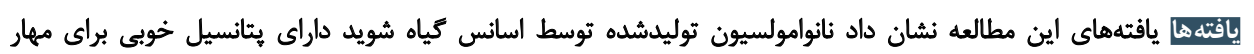

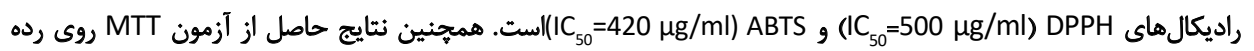

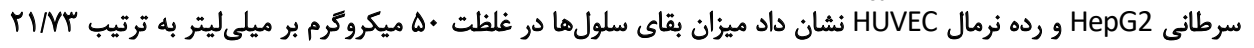

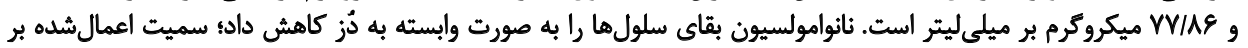

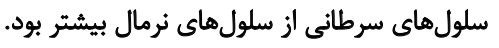

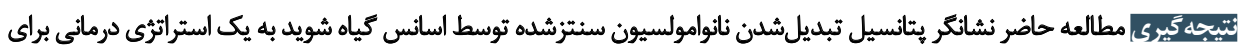
بيمارىهاى ناشى از استرس اكسيداتيو در آينده است
\end{abstract}

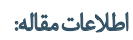

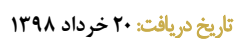

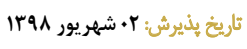

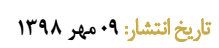

كليدواروها:

نانوامولسيون، كياه

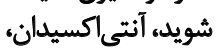
ضدسرطان

به عنوان ناقلين تحويل دارو به سلولهاى سرطانى، توجه زيادى

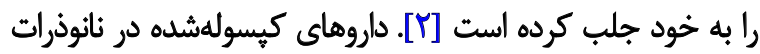

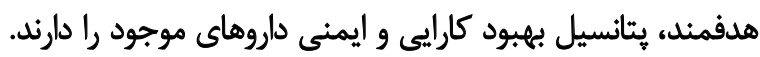

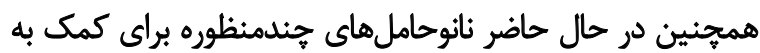

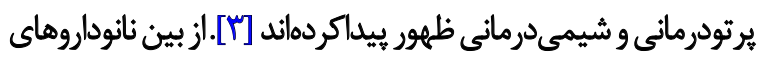

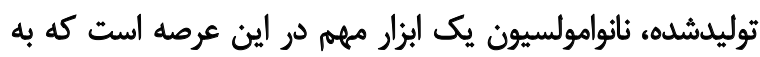

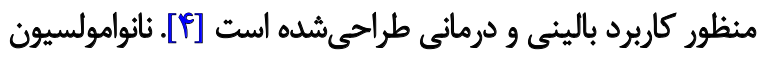

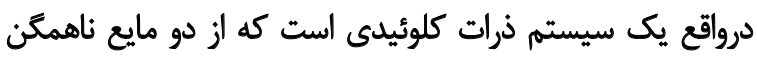

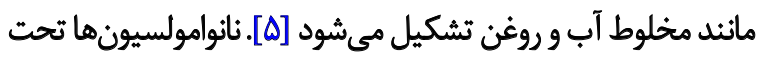

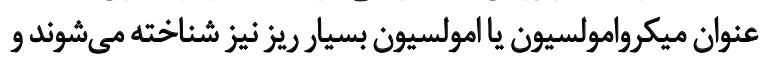

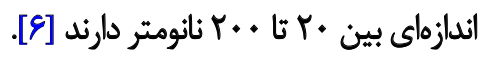

زاستفاده از فناورى نانو براى درمان سرطان، در سال هاى اخير توجه

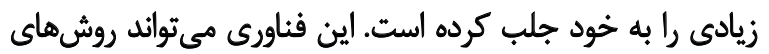

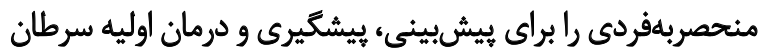

[ارائه كند [1]].

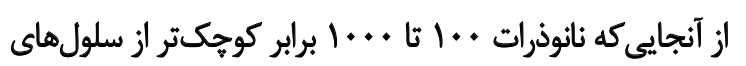

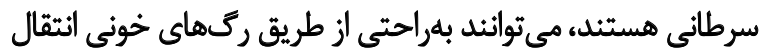

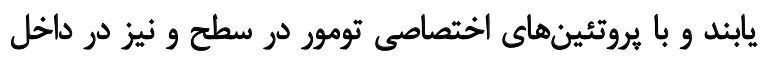

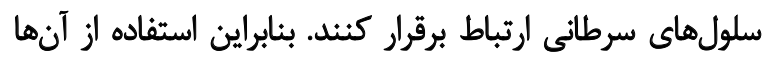




\section{مواد وروشها}

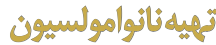

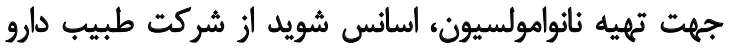

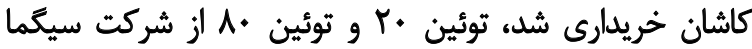

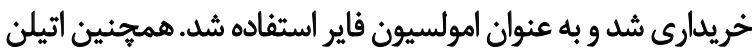

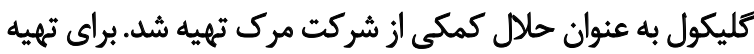

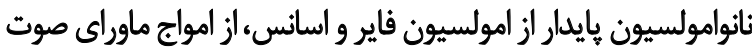

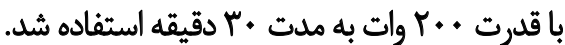

\section{كشيث سلول}

ردهاى سلولى سرطان كبد (HepG2) و اندوتليال سياهرك بند

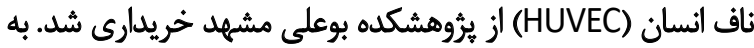

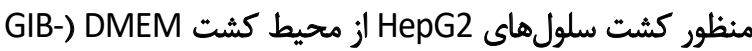
RPMI و براى كشت سلولهاى (CO, USA (GIBCO, USA) RPMI و DMMEM

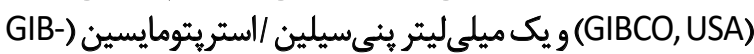
(CO, USA

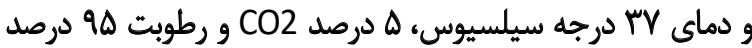

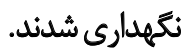

\section{MTT أر أمون}

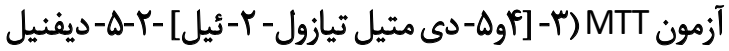

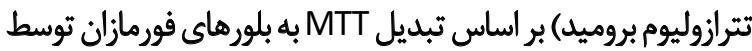

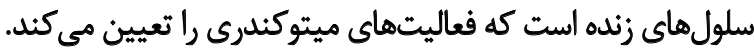

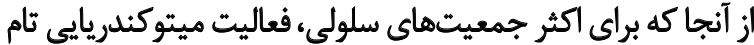

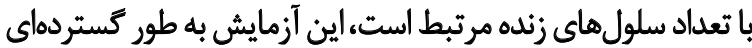

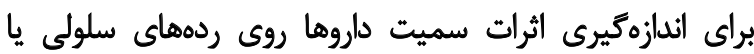

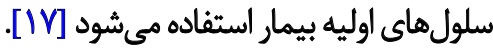

در اين روش سميت نانوامولسيون به منظور مقايسه تأثير آن بر بر بران

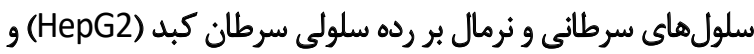

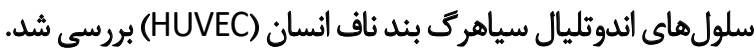

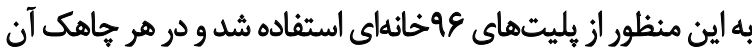

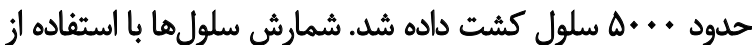

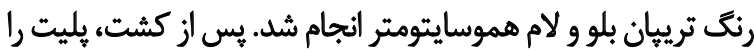

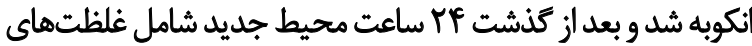

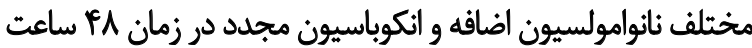

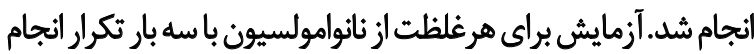

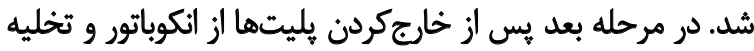

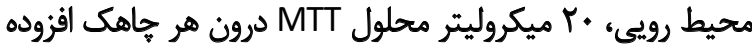

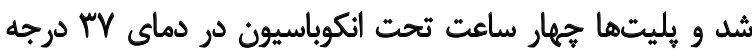
قرار كرفتند. در مرحله بعل محيط كشت و MTT خارج شد و .. 1
در برخى مطالعات نشان داده شده است نانوامولسيونها مزاياى

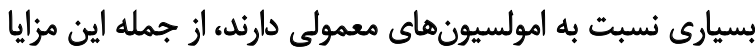

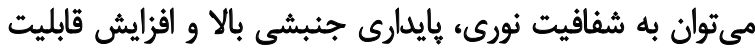

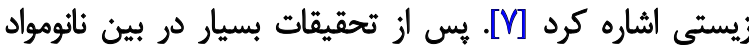

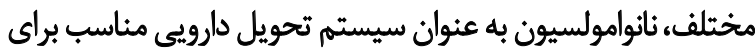

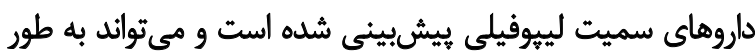
مؤثرى جايكزين شيمىدرمانى باشد.

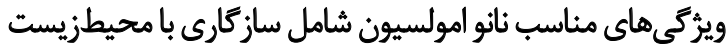

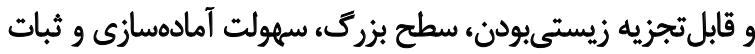

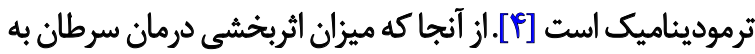

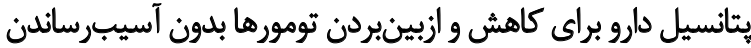

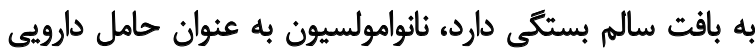

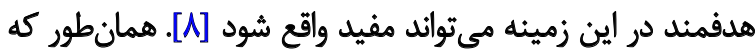

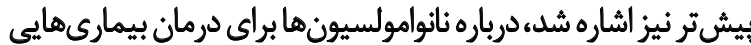

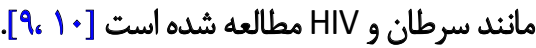

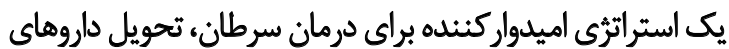

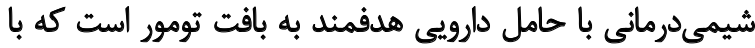

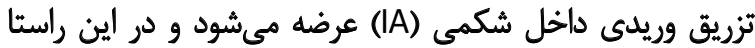

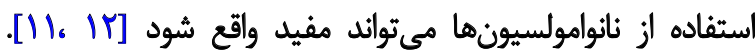

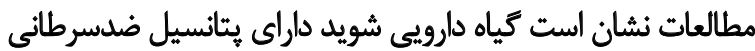

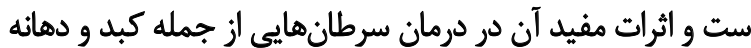

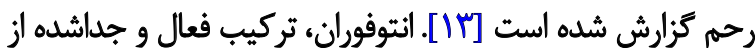

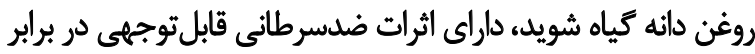

انواع مختلف سرطان است [1F]

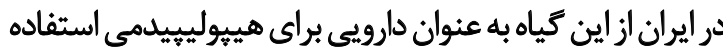

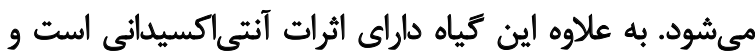

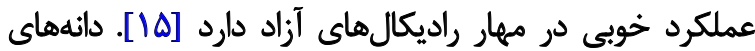

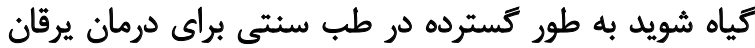

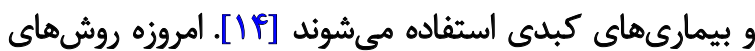

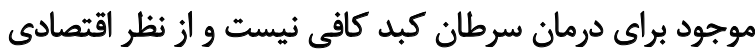

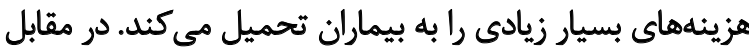

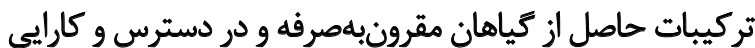

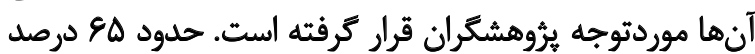

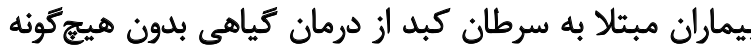

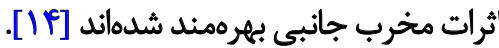

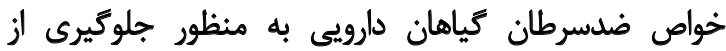

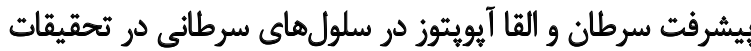

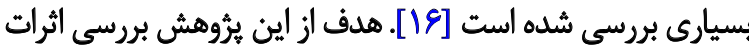

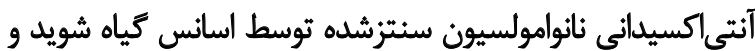

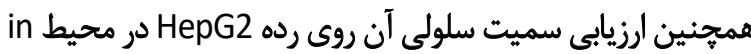
است. vitro 
مدنظر تحت عنوان كلوتاثيون احيا'مخلوط شد. يس از •ب دقيقه،

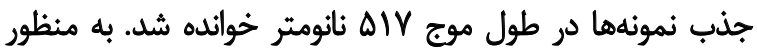

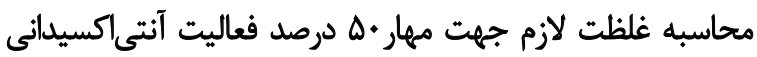

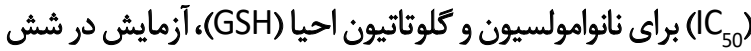

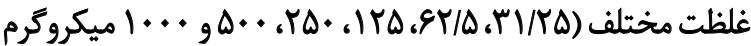

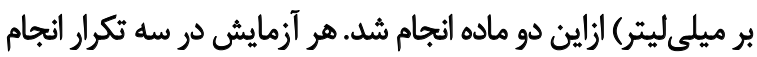

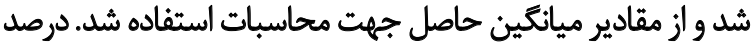
مهار راديكال DPPH به وسيله فرمول شماره Y به دست آمست آمد:

.$r$

DPPH درصد فعاليت راديكالزداييى(A $=100$ آبرقطر)= Aذب شاهد (محلول آماده DPPH و به جاى نانوامولسيون آبمقطر)

A

ABTS أر أمون

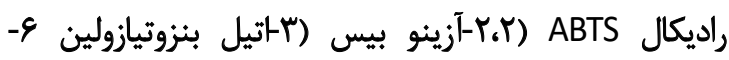

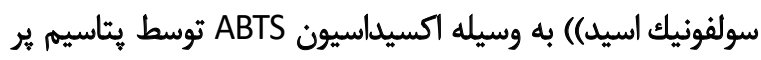

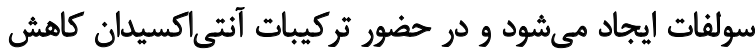

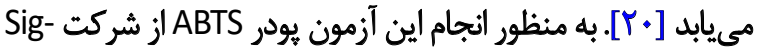
ma-Aldrich, USA

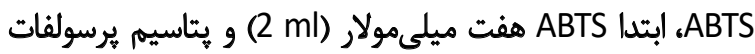
r/FA يرسولفات با يكديكر مخلوط شداند و سيس محلول

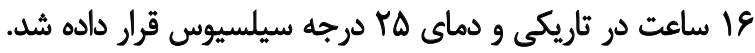

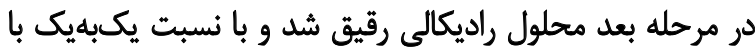

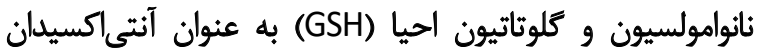

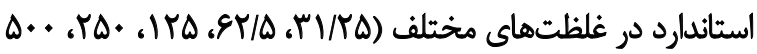

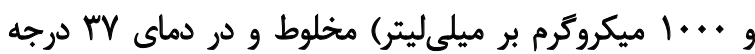

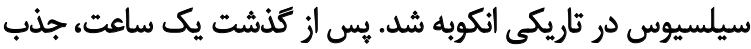

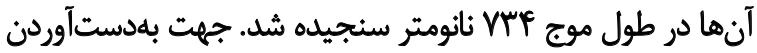

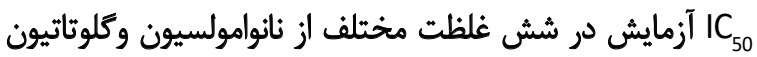

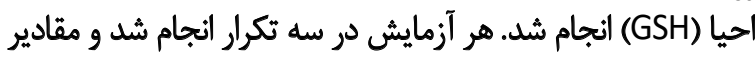

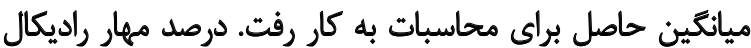

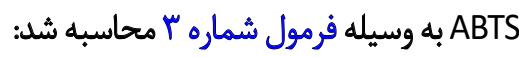

.r

ABTS درصد فعاليت راديكالزدايي $=100$ A آبمقطر)

=جذب نمونه (محلول آماده ABTS و نانوامولسيون)

1. Glutathione (GSH)
ميكروليتر DMSO به هريك از حاهكها اضافه شد. DMSO سبب

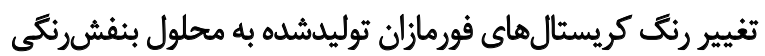
شد ش شدت رنت ايجادشده به وسيله دستًاه

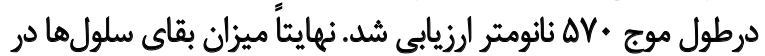
هر غلظت به وسيله فرمول شماره أ محاسبه شداند.

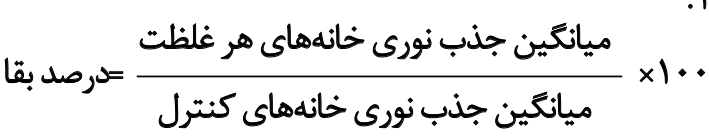

در در فرمول شماره اكنترل نشانكر ميزان جذب نوري فورئ دورمازان

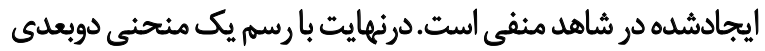

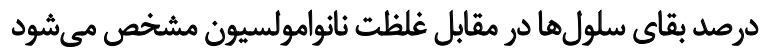

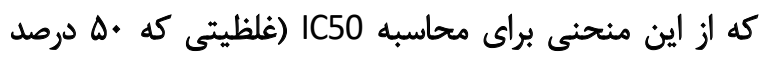
سلولها سالم و •ه درصد سلولها مردهاند) استفاده هي مشود.

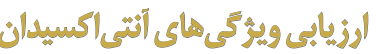

روش رهاى بيىشمارى براي ارزيابى فعاليتهاي آنتىاكسيدانى

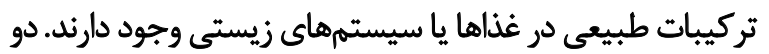

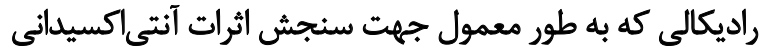

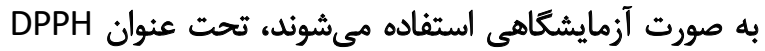

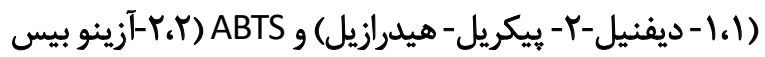

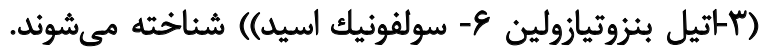

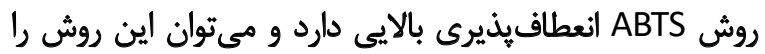

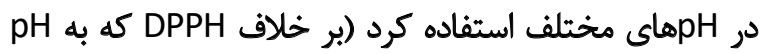

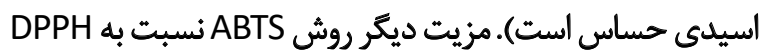

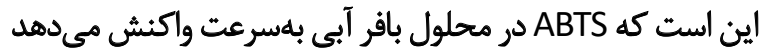

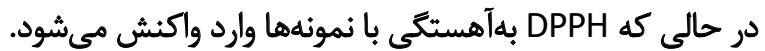

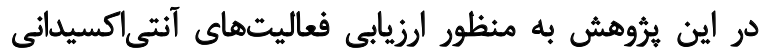
نانوامولسيون توليدشده توسط اسانس كياه شويد از هر دو آزماني

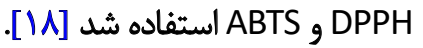

\section{DPPH آن أمون}

DPPH سنجش خاصيت آنتىاكسيدانى بر مبناى انتقال الكترون است كيدي

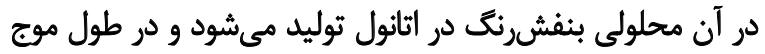

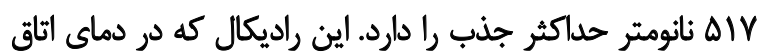

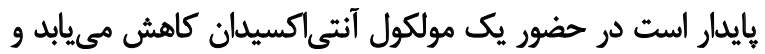

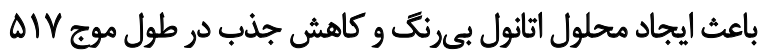
نانومتر ميشود [19] Sigma-Aldrich, از شهت انجام اين آزمون يودر DPPH

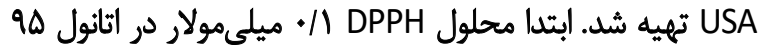

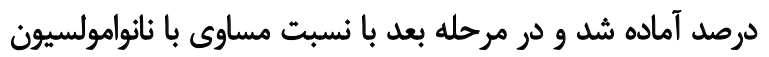
سنتزشده توسط اسانس كياه شويد و تركيب آنتى اكسيدان استاندارد 
با كلوتاتيون به عنوان كنترل مثبت است. همانطور كه ملاحظه

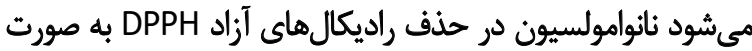

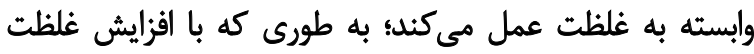

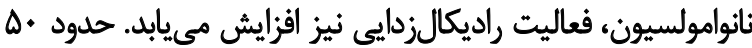

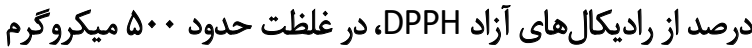

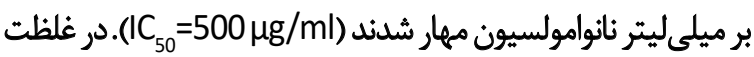

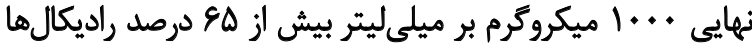
حذف شنف

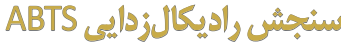

تصوير شماره ب فعاليت حذف راديكال هاى ABTS نانوامولسيون

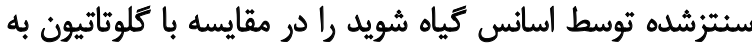

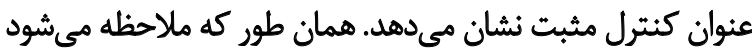

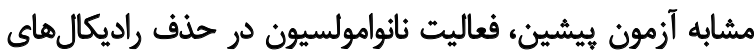

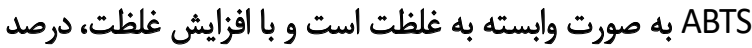

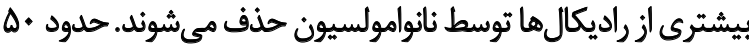

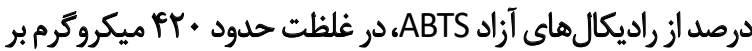
ميلى ليتر نانوامولسيون مهار شدند (IC

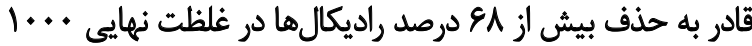

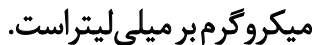
بحث

در مطالعه حاضر، اثرات آنتى|كسيدانى و سميت سلولى

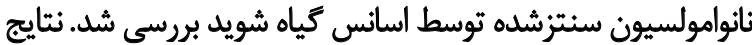

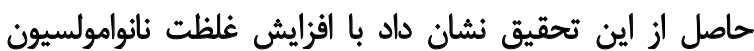

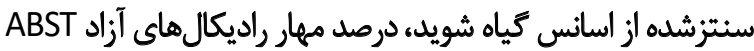

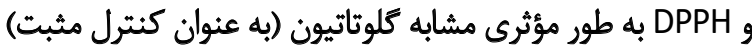

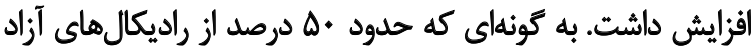

S)

جهت ارزيابي ميزان بقاى سلولهاي تيمارشده و خاصيت

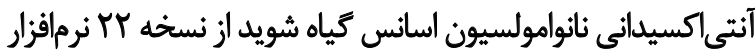
استفاده شد. تحليل واريائس يكطرفه و و مقايسه ميانكينها

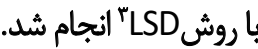

يافتهها

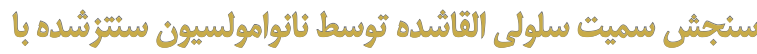

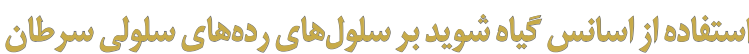

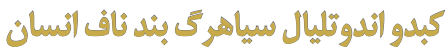

تصوير شماره 1 نشاندهنده سميت سلولى نانوامولسيون

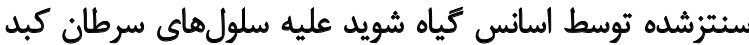

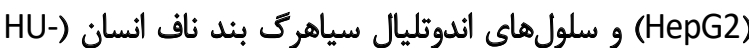

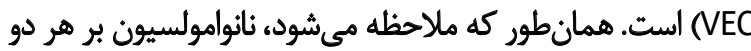

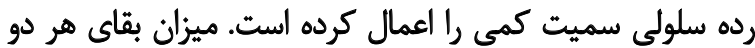

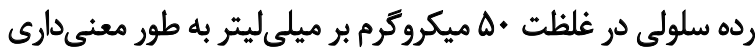

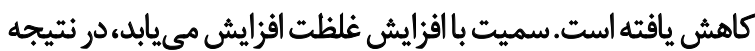

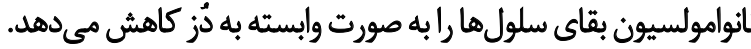
سميت اعمالشده بر سلولهاي سرطانى از سلولهائهائ نرمال بيشتر

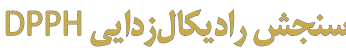

تصوير شماره Y نشاندهنده فعاليت نانوامولسيون سنتزشده توسط اسانس كياه شويد در حذف راديكالهاي آزاد

2. One way ANOVA

3. Least Significant Diffrences
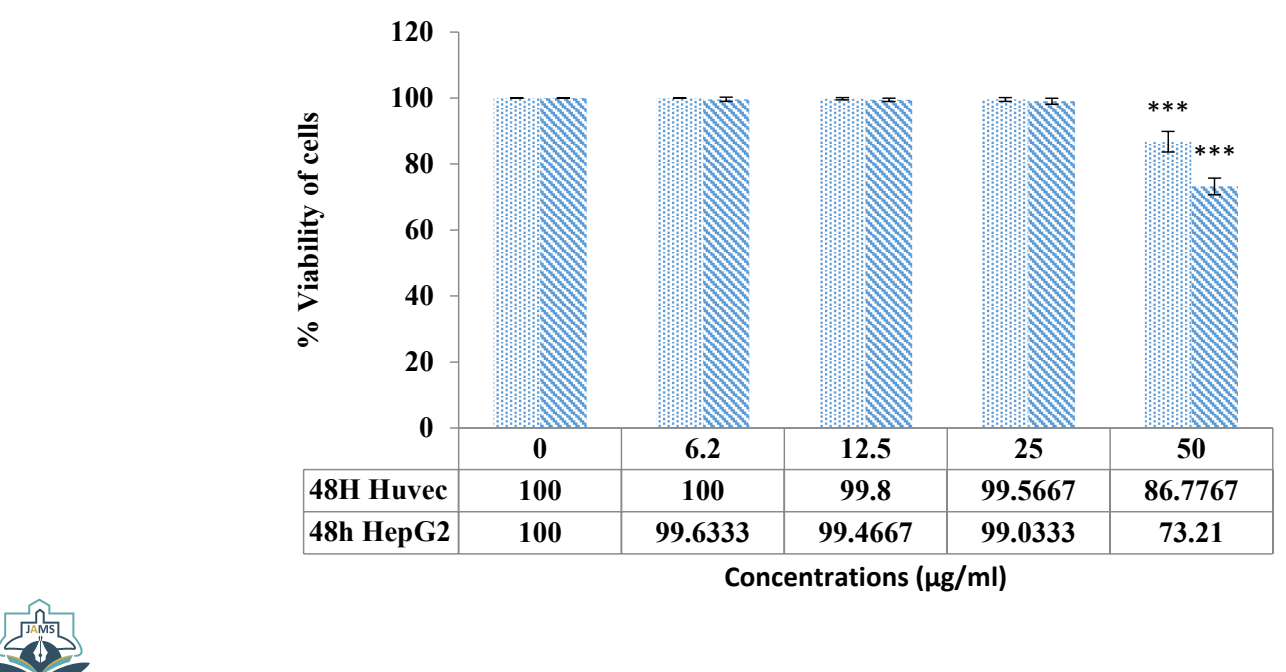

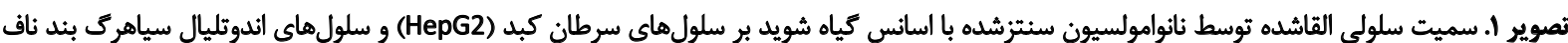

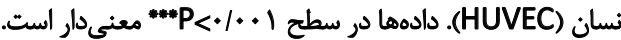




\section{DPPH}

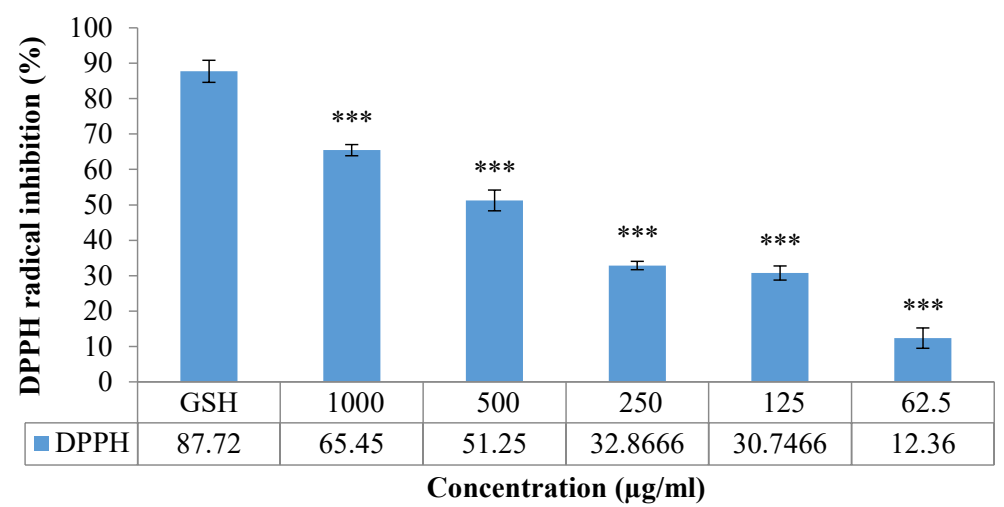

تصوير ז. فعاليت آنتىاكسيداني نانوامولسيون سنتز شده توسط اسائس كياه شويد با استفاده از آزمون DPPH. در اين آزمون كلوتاثيون به عنوان استاندارد استفاده

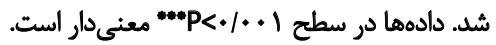

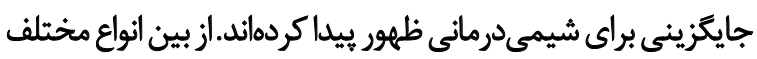

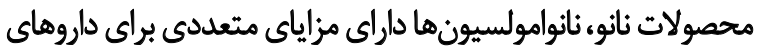

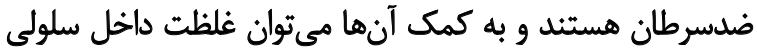

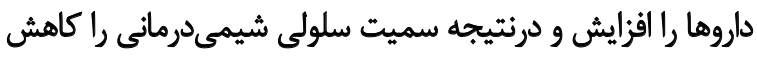

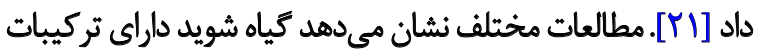

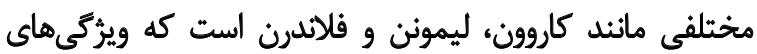

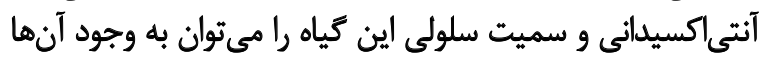

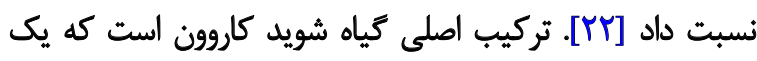
تركيب شيميايي بالقوه براى حفاظت در برابر سرطان

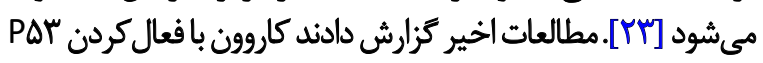

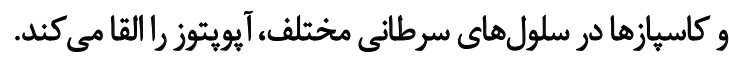
در مطالعهاى مشابه كه توسط مقدسى و همكاران صورت كرفت،

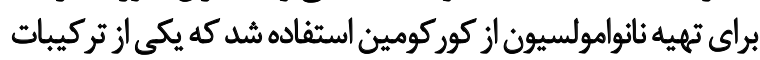

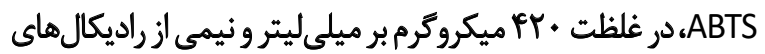

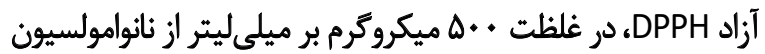

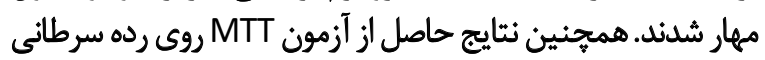

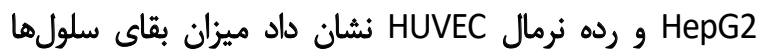

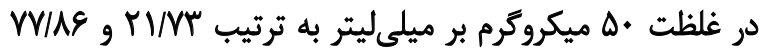

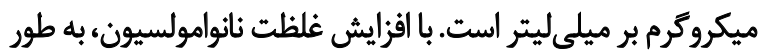

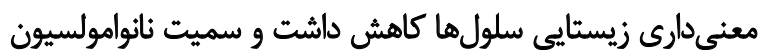

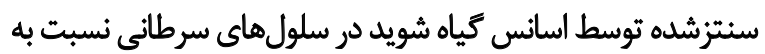

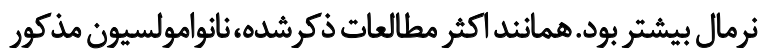

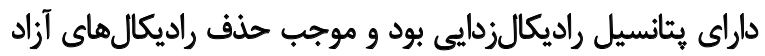

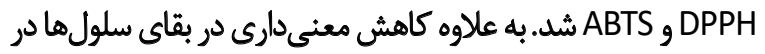

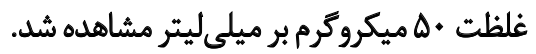

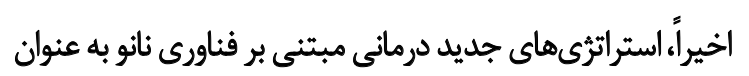

\section{ABTS}

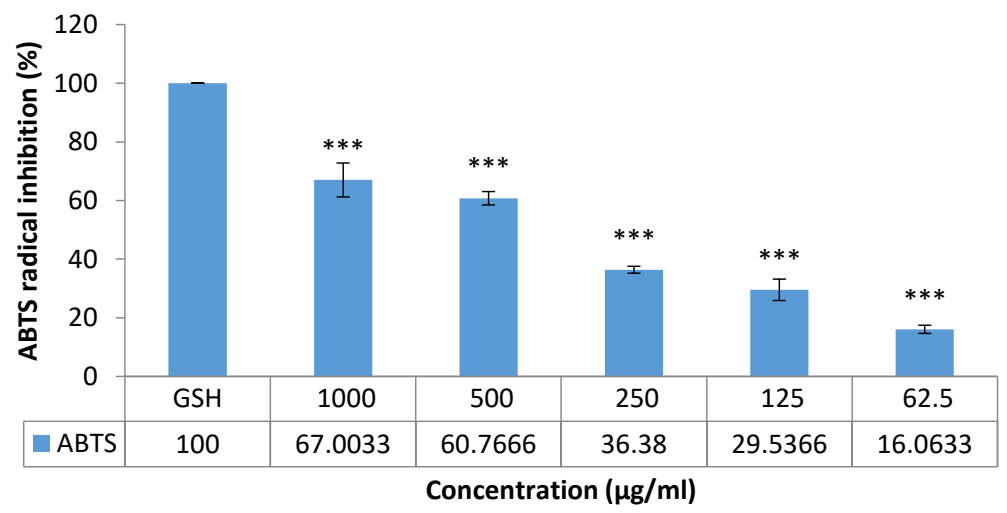

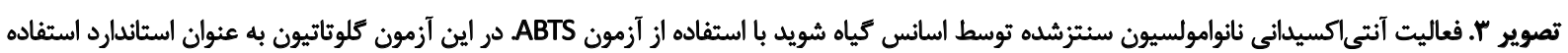

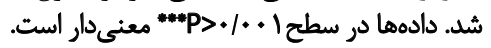


دارويى شويد ارزيابى شد. براى اين منظور از آزمون MTT و رده

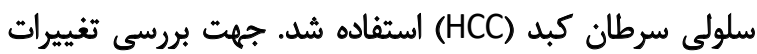
مورفولوزيكى، رنگَآميزى اكريدين (اورنج / اتيديوم برومايد استفاده

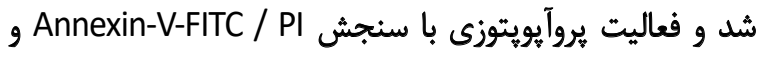

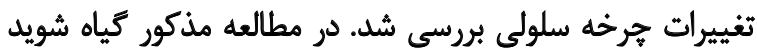

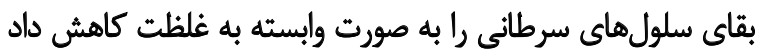

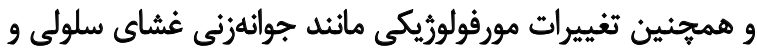

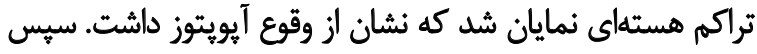

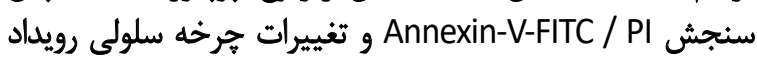

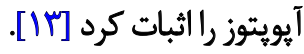

در مطالعهاى كه روى موشهايى با كلسترول بالاصورت كرفت

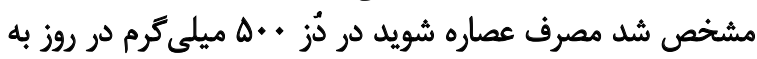

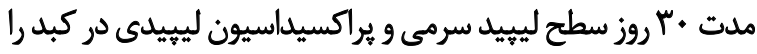

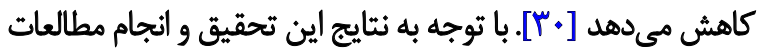

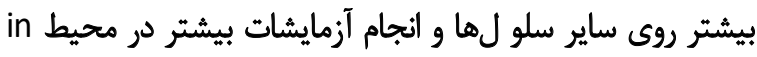

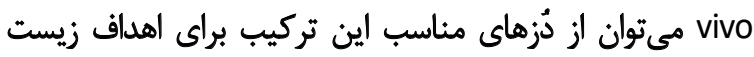

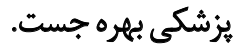

\section{تثيجهيَيرى}

در يُوهشى كه انجام شد، نانوامولسيون سنتزشده توسط اسانس

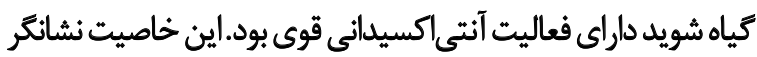

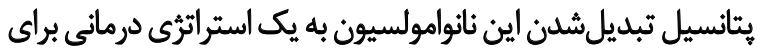

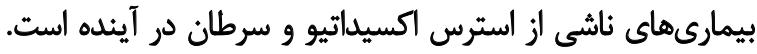

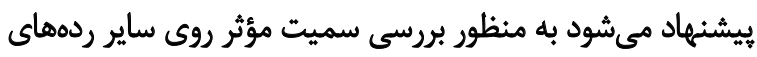

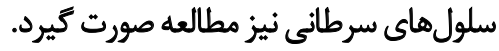

ملاحظات اخلاقى - اتي

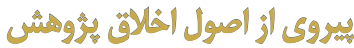

تمامى آزمايشهاي اين تحقيق با رعايت موازين اخلاقى و طبق

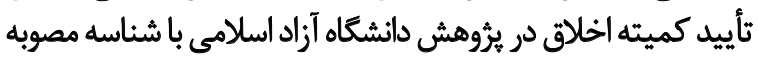

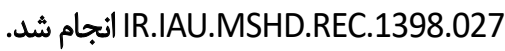

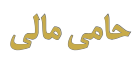

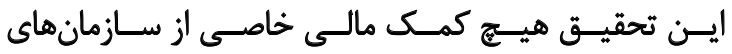

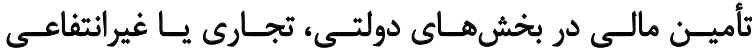

$$
\text { دريافت نكـرده اسـت دئ. }
$$

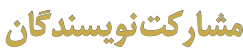

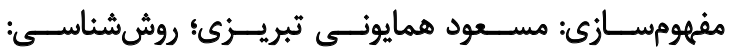

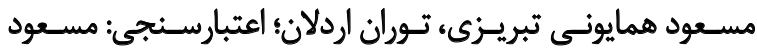

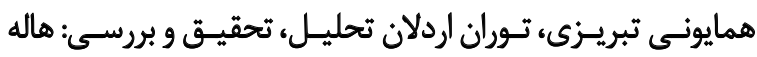

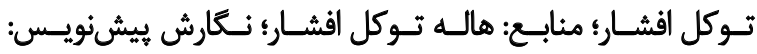

مهم يلىفنوليك است. Nano-CUR در روغن فلفل سياه به عنوان

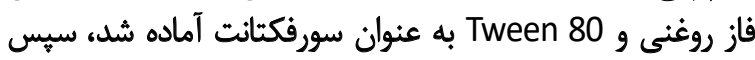
خواص آنتىاكسيدان و سميت آن به كمك آزمونهاى DPPH و

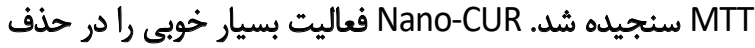

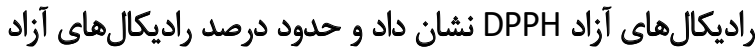

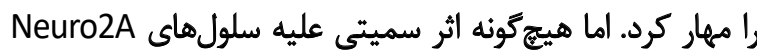

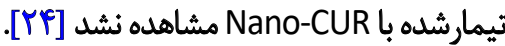

در تحقيقى اثرات آنتىاكسيدانى نانوامولسيون تهيهشده از كياه

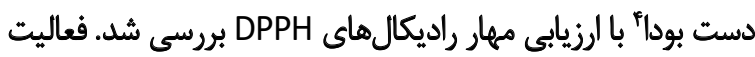

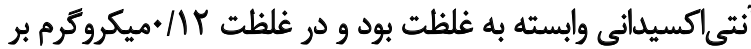

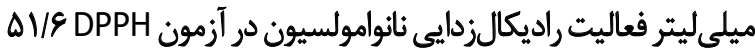

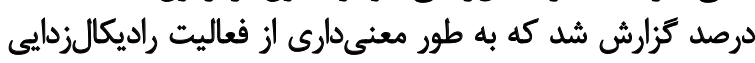

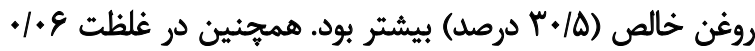

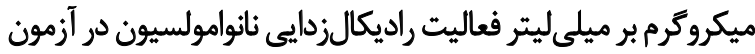

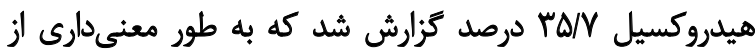

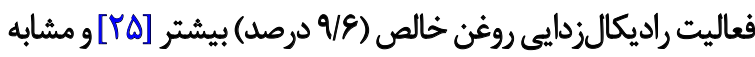

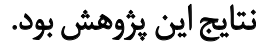

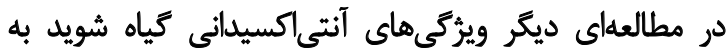

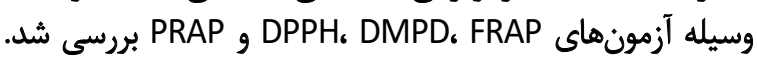

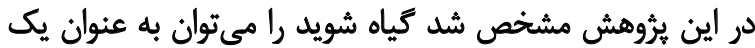

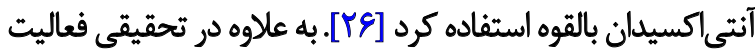

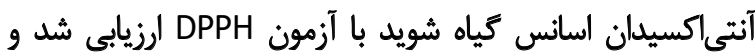

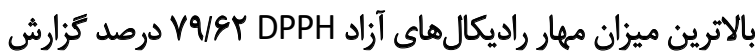

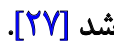

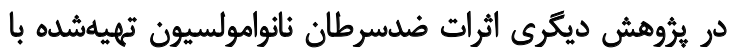

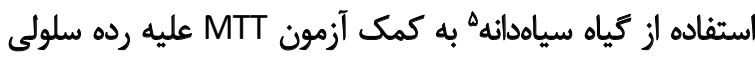

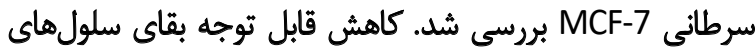

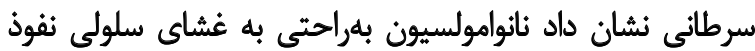

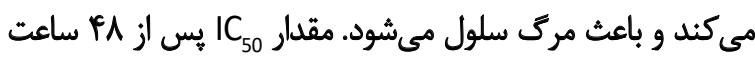

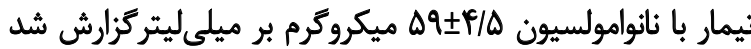

دا درادها مطالعه ديكرى اثرات سميت سلولى نانوامولسيون توليدشده

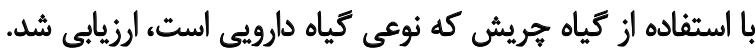

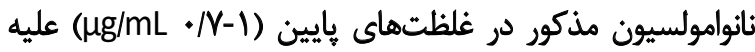

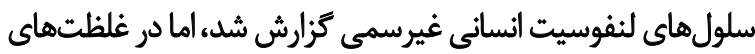

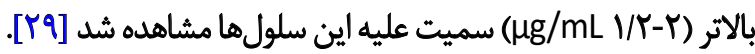

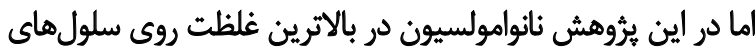
سرطانى كبد سميت كمى ازي خود نشان داد.

علاوه بر مطالعات فوق، در بُروهشى خواص ضدسرطان كياه

4. Citrus medica L. var. sarcodactylis

5. Nigella sativa $L$ 


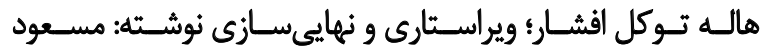

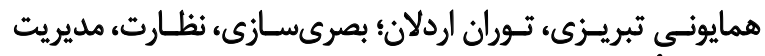

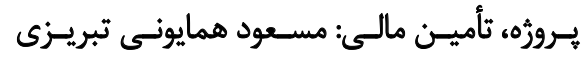

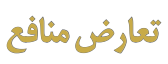

طبق نظر نويسندكان اين مقاله هيجَّونه تعارض منافعى ندارد. 


\section{References}

[1] Misra R, Acharya S, Sahoo SK. Cancer nanotechnology: Application of nanotechnology in cancer therapy. Drug Discov Today. 2010; 15(19-20):842-50. [DOI:10.1016/j.drudis.2010.08.006] [PMID]

[2] Wang X, Yang L, Chen ZG, Shin DM. Application of nanotechnology in cancer therapy and imaging. CA Cancer J Clin. 2008; 58: 97-110. [DOI:10.3322/CA.2007.0003] [PMID]

[3] Alexis F, Rhee JW, Richie JP, Radovic-Moreno AF, Langer R, Farokhzad OC. New frontiers in nanotechnology for cancer treatment. Urologic Oncology. 2008; 26(1):74-85. [DOI:10.1016/j.urolonc.2007.03.017] [PMID]

[4] Sahu P, Das D, Mishra VK, Kashaw V, Kashaw SK. Nanoemulsion: A novel eon in cancer chemotherapy. Mini- Rev Med Chem. 2017; 17(18):1778-92. [DOI:10.2174/1389557516666160219122755] [PMID]

[5] Javadzadeh Y, Bahari L. Therapeutic nanostructures for dermal and transdermal drug delivery. In: Mihai Grumezescu A, editor. Nano-and Microscale Drug Delivery Systems. Amsterdam: Elsevier; 2017. [DOI:10.1016/B978-0-323-52727-9.00008-X]

[6] Priya LB, Baskaran R, Padma VV. Phytonanoconjugates in oral medicine. In: Andronescu E, Mihai Grumezescu A, editors. Nanostructures for oral Medicine. Amsterdam: Elsevier; 2017. [DOI:10.1016/B978-0-323-47720-8.00022-5]

[7] Teo A, Goh KK, Lee SJ. Nanoparticles and nanoemulsions. In Kumar Anal A, Ahmad I, Noomhorm A, editors. Functional foods and dietary supplements: Processing effects and health benefits. Hoboken: John Wiley \& Sons, Ltd. Publication; 2014. [DOI:10.1002/9781118227800.ch15] [PMID] [PMCID]

[8] Praveen Kumar G, Divya A. Nanoemulsion based targeting in cancer therapeutics. Med Chem. 2015; 5(5):272-84. [DOI:10.4172/2161-0444.1000275]

[9] Ostróżka-Cieślik A, Sarecka-Hujar B. The use of nanotechnology in modern pharmacotherapy. In: Mihai Grumezescu A, editor. Multifunctional systems for combined delivery, biosensing and diagnostics. Amsterdam: Elsevier; 2017. [DOI:10.1016/B978-0323-52725-5.00007-1]

[10] Kundu B, Ghosh D, Sinha MK, Sen PS, Balla VK, Das N, et al. Doxorubicin-intercalated nano-hydroxyapatite drug-delivery system for liver cancer: An animal model. Ceram Int. 2013; 39(8):955766. [DOI:10.1016/j.ceramint.2013.05.074]

[11] Jeon MJ, Gordon AC, Larson AC, Chung JW, Kim YI, Kim D-H. Transcatheter intra-arterial infusion of doxorubicin loaded porous magnetic nano-clusters with iodinated oil for the treatment of liver cancer. Biomat. 2016; 88:25-33. [DOI:10.1016/j.biomaterials.2016.02.021] [PMID] [PMCID]

[12] Yu H, Huang Q. Investigation of the cytotoxicity of foodgrade nanoemulsions in Caco-2 cell monolayers and HepG2 cells. Food Chem. 2013; 141(1):29-33. [DOI:10.1016/j.foodchem.2013.03.009] [PMID]

[13] Mohammed FA, Elkady Al, Syed FQ, Mirza MB, Hakeem KR Alkarim S. Anethum graveolens (dill)-A medicinal herb induces apoptosis and cell cycle arrest in HepG2 cell line. J Ethnopharmacol. 2018; 219:15-22. [DOI:10.1016/j.jep.2018.03.008] [PMID]

[14] Mohammed FA, Razvi SS, Abdul WM, Mohammed K, Hakeem $K R$, Banaganapalli $B$, et al. Protective role of medicinal herb anethum graveolens (Dill) against various human diseases and metabolic disorders. In: Ozturk M, Hakeem KH, editors. Plan and Human Health. Berlin:Springer; 2019. [DOI:10.1007/978-3030-04408-4_8]

[15] Oshaghi EA, Tavilani H, Khodadadi I, Goodarzi MT. Dill tablet: A potential antioxidant and anti-diabetic medicine. Asian Pac J Trop Biomed. 2015; 5(9):720-7. [DOI:10.1016/j.apjtb.2015.06.012]

[16] Safarzadeh E, Shotorbani SS, Baradaran B. Herbal medicine as inducers of apoptosis in cancer treatment. Adv Pharm Bull. 2014; (Suppl. 1) 4(5):421-7. [DOI:10.5681/apb.2014.062] [PMID] [PMCID]

[17] Van Meerloo J, Kaspers GJ, Cloos J. Cell sensitivity as says: The MTT assay. Methods Mol Biol. 2011; 731:237-45. [DOI:10.1007/978-1-61779-080-5_20] [PMID]

[18] Shalaby EA, Shanab SM. Comparison of DPPH and ABTS assays for determining antioxidant potential of water and methanol extracts of Spirulina platensis. Indian J Geo-Marine Sci. 2013; 42(5):556-64.

[19] Garcia EJ, Oldoni TL, Alencar SM, Reis A, Loguercio AD, Grande $\mathrm{RH}$. Antioxidant activity by DPPH assay of potential solutions to be applied on bleached teeth. Braz Dent J. 2012; 23(1):22-7. [DOI:10.1590/S0103-64402012000100004] [PMID]

[20] Re R, Pellegrini N, Proteggente A, Pannala A, Yang M, Rice-Evans C. Antioxidant activity applying an improved ABTS radical cation decolorization assay. Free Radical Bio Med. 1999; 26(9-10):12317. [DOI:10.1016/S0891-5849(98)00315-3]

[21] Severino P, Fangueiro JF, Ferreira SV, Basso R, Chaud MV, Santana $\mathrm{MH}$, et al. Nanoemulsions and nanoparticles for non-melanoma skin cancer: Effects of lipid materials. Clin Transl Oncol. 2013; 15(6):417-24. [DOI:10.1007/s12094-012-0982-0] [PMID]

[22] Yazdanparast R, Bahramikia S. Evaluation of the effect of anethum graveolens $\mathrm{L}$. crude extracts on serum lipids and lipoproteins profiles in hypercholesterolaemic rats. Darg J Pharm Sci. 2008; 16(2):88-94.

[23] Zheng GQ, Kenney PM, Lam LK. Anethofuran, carvone, and limonene: Potential cancer chemoprotective agents from dill weed oil and caraway oil. Planta Medica. 1992; 58(4):338-41. [DOI:10.1055/s-2006-961480] [PMID]

[24] Moghaddasi F, Housaindokht MR, Darroudi M, Bozorgmehr $M R$, Sadeghi A. Synthesis of nano curcumin using black pepper oil by $\mathrm{O} / \mathrm{W}$ nanoemulsion technique and investigation of their biological activities. LWT. 2018; 92:92-100. [DOI:10.1016/j. lwt.2018.02.023

[25] Lou Z, Chen J, Yu F, Wang H, Kou X, Ma C, et al. The antioxidant antibacterial, antibiofilm activity of essential oil from Citrus medica L. var. sarcodactylis and its nanoemulsion. LWT. 2017; 80:3717. [DOI:10.1016/j.lwt.2017.02.037]

[26] Orhan IE, Senol FS, Ozturk N, Celik SA, Pulur A, Kan Y. Phytochemical contents and enzyme inhibitory and antioxidant properties of Anethum graveolens L.(dill) samples cultivated under organic and conventional agricultural conditions. Food Chem Toxicol. 2013; 59:96-103.[DOI:10.1016/j.fct.2013.05.053] [PMID]

[27] Stanojevic L, Stanković MZ, Cvetković D, Danilović B, Stanojević J. Dill (anethum graveolens L.) seeds essential oil as a potential natural antioxidant and antimicrobial agent. Biologica Nyssana. 2016; 7(1):31-9.

[28] Periasamy VS, Athinarayanan J, Alshatwi AA. Anticancer activity of an ultrasonic nanoemulsion formulation of Nigella sativa $\mathrm{L}$. 
essential oil on human breast cancer cells. Ultrason Sonochem. 2016; 31:449-55.[DOI:10.1016/j.ultsonch.2016.01.035] [PMID]

[29] Jerobin J, Makwana P, Kumar RS, Sundaramoorthy R, Mukherjee A, Chandrasekaran N. Antibacterial activity of neem nanoemulsion and its toxicity assessment on human lymphocytes in vitro. Int J Nanomedicine. 2015; 10(1):77-86. [DOI:10.2147/IJN.S79983] [PMID] [PMCID]

[30] Meriem T. Chemical Composition And Anti-inflammatory Activity Of Myrtus Communis L. Essential Oil, Phytothérapie. Algerian J Arid Environ. 2016; 6(2):1-10. 